\title{
Sources and Fate of Microplastics in Urban Areas: A Focus on Paris Megacity
}

\author{
Rachid Dris, Johnny Gasperi, and Bruno Tassin
}

\begin{abstract}
Since the beginning of the 2010s, the number of investigations on microplastics in freshwater increased dramatically. However, almost no study aims at investigating the various sources and fate of microplastics in a catchment. This chapter aims at analyzing the various sources and fate of microplastics for an urban catchment and its hydrosystem (sewage, runoff, etc.). It presents the results obtained during a 3-year study of the Paris Megacity. Such a study required the development of appropriate sampling strategies for each compartment. It was highlighted that fibers are highly concentrated in the studied area, and therefore a focus in this category of microplastics was carried out. The atmospheric fallout exhibited important levels of fibers. However, at the scale of the Parisian agglomeration, wastewater treatment plant disposals and combined sewer overflows represent the major sources (number of fibers introduced per year) among the studied ones.
\end{abstract}

Keywords Fibers, Freshwater, Microplastics, Plastic pollution, Urban areas, Urban impact

\section{Introduction}

Although the first scientific articles that identified plastic in the environment as an issue are rather old [1,2], efforts of the scientific community on this subject actually started at the beginning of the twenty-first century with the 2004 paper of Thompson et al. [3]. Studies highlighted the issue of microplastics (MPs) and raise two main questions: (1) the interaction between plastic items and the

R. Dris $(\triangle)$, J. Gasperi, and B. Tassin

LEESU, UMR MA 102, École des Ponts, UPEC, AgroParisTech, UPE, Paris, France

e-mail: rachid.dris@enpc.fr 
physical-chemical environment (fragmentation, micro-pollutant exchanges) and (2) their interaction with the biological compartment including ecotoxicological effects but also biodegradation. As underlined by Dris et al. [4], the term "microplastics" was used first in the Thompson article to describe mainly plastic particles that are "fibrous, $20 \mu \mathrm{m}$ in diameter, and brightly colored." However, in 2008 another definition of microplastics with a much broader scope was proposed that included all the particles with a size smaller than $5 \mathrm{~mm}$ [5]. Although, on the basis of the usual scientific meaning of "micro," microplastics will describe "micrometric" particles (i.e., 1-1,000 $\mu \mathrm{m}$ ), for the scientific community, the name

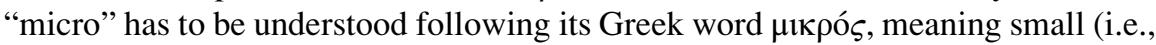
all particles $<5 \mathrm{~mm}$ ).

This issue of microplastics in the environment has received increasing attention from the scientists, and the number of articles on this subject increased from less than 10 in 2011 to more than 150 in 2016 (request on Scopus with the words "microplastic" and "environment"). However, microplastics in freshwater environments represent only a small fraction of this amount. The first articles on microplastics in freshwater were published in 2011 and focus mainly on Lake Huron [6] and Los Angeles rivers [7] in the USA. Since that period, numerous studies have been published covering all continents, with the exception of Antarctica, and all the potentially impacted environments (water and sediment for the aquatic environment, banks for the terrestrial environment). While at the beginning mainly lakes have been investigated, it can be considered that now both lentic and lotic ecosystems are investigated. Nevertheless, the dynamics of fibers and plastics in urban catchments and hydrosystems are practically unknown. Their fate, transfer routes, and processes in continental water have yet to be determined.

Cities can be considered as one of the major sources of MPs as they gather at an especially high density all the activities that involve plastics and MPs including textile uses, packaging, transportation, electronics, buildings, and constructions. The aim of this work is to provide a comprehensive analysis of the sources and fate of microplastics in an urban environment with a focus on the Paris Megacity. Often referred to as the Paris agglomeration, this megacity is one the world's 40 largest with a population of over 10 million. The Paris agglomeration is crossed by the Seine River, whose catchment drains an area of approximately $32,000 \mathrm{~km}^{2}$ from the river's headwaters to Paris. This catchment combines intense anthropogenic pressures with a very limited dilution factor due to the low average flow of the Seine River $\left(350 \mathrm{~m}^{3} \mathrm{~s}^{-1}\right.$ in Paris). As a consequence, the Paris agglomeration exerts a dramatic pressure regarding pollutants on the river and provides a good case study in order to understand the consequences of urban environments on continental water. Moreover, to our knowledge, there is no case study dealing with MPs and covering all the compartments of the urban system and fluxes that occur between them. This study on Paris Megacity provides, from our point of view, the most comprehensive overview of MP sources in an urban area and its interaction with continental water. 


\section{Types and Shapes of Microplastics: A Focus on Fibers in Urban Areas?}

MPs observed in freshwater not only cover several orders of magnitude in size, they also cover a wide spectrum of shapes. This includes fibers (length $\gg$ diameter) and fragments (diameter $\gg$ thickness) composed of different irregular shapes and spheres. These can be considered as either primary or secondary MPs depending on their origin.

Primary MPs are already manufactured in a size smaller than $5 \mathrm{~mm}$. Two different forms of primary MPs exist: preproduction pellets and microbeads. Preproduction pellets are used in plastic industry. These virgin resins are melted and then formed into consumer products [8]. Microbeads were first present in hand cleaners that are used on rare occasion by the average consumer [9]. Microbeads have also come to replace natural exfoliating materials in facial cleansers, which are often used on a daily or at least weekly basis [10]. Secondary MPs stem from the degradation and fragmentation of large debris. Thermal, mechanical, and photodegradation are important factors during the fragmentation process $[11,12]$.

We consider textile fibers to represent a special case. They can be considered as secondary MPs as they come from the breakdown of large items (clothes). This breakdown does not primarily occur in the environment but in the washing machines during the laundry [13]. As a consequence, fibers are found in the disposal of washing machines and, like primary MPs, enter the environment in a microscopic size. The same study showed the presence of fibers at the disposal of wastewater treatment plants (WWTPs). As a consequence, we would expect a predominance of fibers in urban areas with large WWTPs. In addition, because of the complexity of studying fibers, they are often overlooked. It is here decided as consequence to give a specific attention to fibers.

Fibers are often not included on the key figures concerning plastic materials [14]. However, a great proportion of the produced fibers are derived from petrochemical polymers. The international organization for standardization (ISO/TR 11827:2012 Textiles - Composition testing - Identification of fibers) proposed a classification of the fibers according to their nature and origin.

Fibers that are used and commercialized can be either natural or man-made. The natural fibers are categorized according to their origin into animal, vegetal, or mineral fibers. For instance, cotton is a vegetal natural fiber and is very widely used. Man-made fibers are obtained by a manufacturing process. The artificial ones are made by the transformation of natural polymers. For example, rayon is artificially manufactured but is made from cellulose, which is a natural polymer. On the other side, synthetic fibers are made from polymers that were chemically synthetized. In this latter category, we can find plastic polymers (polypropylene, PP; polyamide, PA; polyether sulfone, PES; etc.). The latter are most often the only fibers that are included in the microplastics definition in the different studies. Bicomponent fibers also exist and are composed of two fibers forming polymer components, which are chemically and/or physically different. 
In 2014, a total of 91 million metric tons of textile fibers were produced [15], of which 63 million metric tons were man-made fibers and 58 million metric tons were plastic fibers $(\approx 20 \%$ of the world's plastic production).

\section{Source and Fate of Microplastics on the Paris Megacity}

\subsection{Overview of the Approach}

Between 2014 and 2016, an investigation of several sources and fluxes of MPs has been carried out on the Paris Megacity. The following sources have been investigated:

1. Atmospheric fallout

2. Runoff water

3. Gray water

4. Wastewater and WWTP outlets

5. Combined sewer overflows (CSOs)

A map of Paris Megacity as well as the location of the various sampling sites is presented (Fig. 1). More details on the methodology and the approach can be found in Dris [16-18].

One of the challenges of a holistic study is that the different sample types will require different sampling methods. For instance, atmospheric fallout requires the use of a funnel for sampling, while automatic samplers including pumping devices are needed for the inaccessible canals of wastewater and CSOs. The other difficulty for such a systemic approach is the interpretation and comparison of the results. The atmospheric fallout is intrinsically expressed differently than the other compartments (fibers per surface and time unit rather than a concentration). Moreover, the interpretation has to take into consideration the time and space scale differences among the compartments. For instance, atmospheric fallout is a diffusive permanent source, whereas WWTP outlets represent a punctual but permanent source, while CSOs are punctual in time and space. The best way to work around this and be able to understand the sources of MPs is to consider the fluxes between the various compartments, for instance, on an annual basis. In this chapter, a first attempt to describe this approach is presented (Table 1).

\subsection{Microplastics Encountered in the Different Compartments}

The various concentrations of fibers and fragments encountered in each compartment are synthetized in Fig. 2. The total atmospheric fallout was investigated at two 


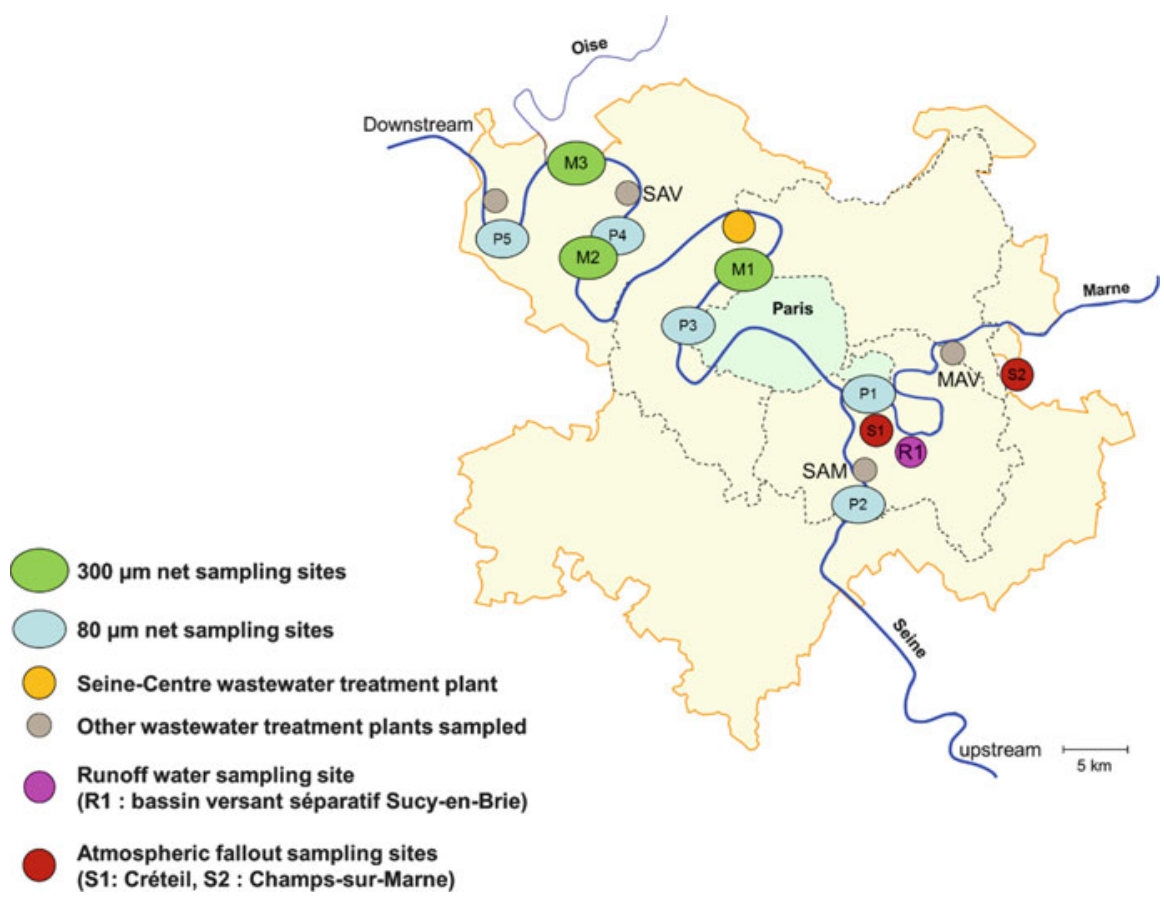

Fig. 1 Map of Paris Megacity and location of the various sampling sites [23]

Table 1 Used methods and sampled volumes for the various compartments

\begin{tabular}{l|l|l}
\hline & Sampling method & Sampled volume \\
\hline Atmospheric fallout & A collection funnel & Continuous monitoring \\
\hline Urban runoff, WWTP effluents, CSOs & Automatic samplers & $200-1,500 \mathrm{~mL}$ \\
\hline Fibers in freshwater & $80 \mu \mathrm{m}$ mesh size net & $0.2-4 \mathrm{~m}^{3}$ \\
\hline Fragments in freshwater & $300 \mu \mathrm{m}$ mesh size net & $50-200 \mathrm{~m}^{3}$ \\
\hline
\end{tabular}

sampling sites: one in a dense urban environment and one in suburban environment [17]. Only fibers were encountered and fragments were not detected. Throughout the year of monitoring (site 1), the atmospheric fallout ranged from 2 to 355 fibers $\mathrm{m}^{-2}$ day $^{-1}\left(110 \pm 96\right.$ fibers $\mathrm{m}^{-2}$ day $^{-1}$, mean $\left.\pm \mathrm{SD}\right)$, indicating a high annual variability. On site 2 , the atmospheric fallout was estimated around $53 \pm 38$ fibers $\mathrm{m}^{-2}$ day $^{-1}$. When the levels on both sites are compared, the suburban site systematically showed fewer fibers than the urban one. We hypothesize that this difference can be explained by the density of the surrounding population, which is considered as a proxy of local activity. However, this has to be confirmed through investigations at other sites.

A suburban catchment with a separate sewer (wastewater and runoff water are collected separately) was considered for the urban runoff (unpublished data). The Sucy-en-Brie (R1, Fig. 1) catchment area reaches 261 ha, and its impervious 

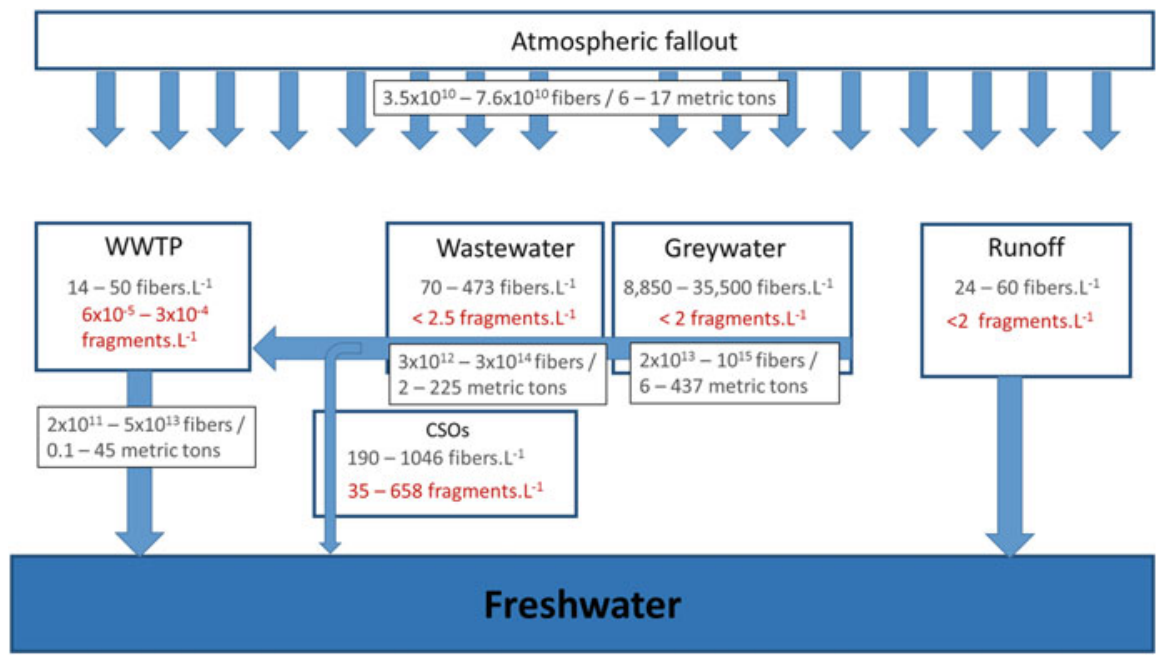

Fig. 2 A synthesis of the total fibers and fragment concentrations in the investigated urban compartments as well as the fluxes of synthetic fibers between different compartments. Only data about total fibers (natural fiber like cotton + artificial and synthetic fibers) are available for CSOs and runoff, and therefore the latter is not included in the synthetic fiber flux estimations [24]

surface coefficient is about $27 \%$. At the outlet of this catchment, stormwater was collected during five different precipitation events between October 2014 and March 2016. Runoff concentrations at the catchment outlet ranged between 24 and 60 fibers $\mathrm{L}^{-1}$ with an average of 35 fibers $\mathrm{L}^{-1}$. By considering the rainwater volumes collected during the atmospheric fallout experiments, a median concentration of 40 fibers $\mathrm{L}^{-1}$ of rainwater can be estimated. Fragments of plastic were also recovered in the runoff on three of the five sampled events and consisted mainly of foamy and irregular shapes. Concentrations ranged from $<2$ to 16 fragments $\mathrm{L}^{-1}$. The number of fragments was systematically lower than the number of fibers, and there is no correlation between both types of MPs.

MPs were also analyzed at various scales of the sewer system: from the outlet of washing machines to the outlet of WWTPs (unpublished data except for few samples of one WWTP outlet [18]). Washing machine effluents have been collected at four volunteers' homes. The washings were carried out in their own washing machines with their own items. To have a more realistic vision, participants were asked to not change anything from their habitual washing process. Therefore, clothes made from natural, artificial, and synthetic fibers were all included, without any preselection. Concentrations between 8,850 and 35,500 fibers $\mathrm{L}^{-1}$ were encountered compared to 120 and 810 fibers $\mathrm{L}^{-1}$ for control washes (without clothes), confirming the large amount of the fibers disposed of in washing machines' effluents.

The wastewater entering four WWTPs of the Paris agglomeration was considered. Generally, wastewater is highly contaminated with fibers with the average concentration being of $248 \pm 109$ fibers $\mathrm{L}^{-1}$ (mean $\pm \mathrm{SD}, n=20$ ) reaching a 
maximum of 473 fibers $\mathrm{L}^{-1}$. In comparison, another study found similar values about 180 textile fibers $\mathrm{L}^{-1}$ [19].

Unlike fibers, fragments were only rarely collected, probably because of the low volumes of the samples (100-400 mL). In the 20 samples studied, 16 did not contain any fragments. For the four remaining samples, three contained one fragment, while the other contained two fragments. These small values indicate a potential upper limit for the concentration of these particles. No fragments in a $400 \mathrm{~mL}$ sample, for instance, imply a concentration $<2.5$ particles $\mathrm{L}^{-1}$. Larger values were observed in other studies: 430 particles $\mathrm{L}^{-1}$ [19] or 13 particles $\mathrm{L}^{-1}$ [20]. If this variation in fragment concentrations is site dependent or is related to different methodologies, it remains unknown at the moment. Fragments could, in an oversized sewage network, settle inside the sewer during dry weather periods and reach the WWTP only at low concentrations. It is known that Paris wastewater sewerage system is oversized leading to a very low flow velocity and therefore a high sedimentation of particles inside the sewer.

Two WWTPs were selected in order to estimate the treatment efficiency. Seine Centre WWTP was considered for the case of a treatment by biofilters, while Seine Aval WWTP is a conventional activated sludge plant. For Seine Centre, three sampling campaigns on three consecutive working days were carried out. Raw wastewater after pretreatment, settled wastewater (after the primary clarifier), and treated water (after the biofilters treatment) were all considered. Only limited volumes were considered here. As the disposal channel of Seine Aval is large and long enough to be navigable, a manta trawl with a $330 \mu \mathrm{m}$ mesh size was towed by a motor boat in the upstream direction in order to sample high volumes $\left(68 \mathrm{~m}^{3}\right)$ and count fragments.

In the case of Seine Centre, only fibers were encountered. The results suggested a removal from 83 to $95 \%$ of the fiber contamination. Other works showed higher removal efficiencies of MPs by WWTPs, i.e., between 95 and 99\% [20, 21]. However, all these results are hardly comparable since methods used vary from one study to another.

Fragments were detected at the outlet of the WWTP Seine Aval because larger volumes were integrated. Irregular fragments were observed primarily, and foams, films, and spheres were found punctually. The number of fragments observed in the treated water varies between $6 \times 10^{-5}$ and $3 \times 10^{-4}$ fragments $\mathrm{L}^{-1}$. The concentrations of fragments are much lower than the levels of fibers observed in the automatic sampler samples $\left(10^{5}\right.$ more fibers). Being able to observe fragments in the manta trawl samples is also an additional indication that fragments are present in WWTP effluents but just too scarce to be observed in small sample volumes.

A combined sewer system collects rainwater runoff and domestic sewage. However, during heavy rainfall events, the volume of water may exceed the capacity of the sewer and can cause flood events. In this situation, untreated water is discharged directly into the water bodies. In this context, $500 \mathrm{~mL}$ samples were collected at La Briche CSO outlet during three events.

Both fragments and fibers were encountered in the CSO samples. For fibers, levels of 190,898 and 1,046 fibers $\mathrm{L}^{-1}$ were detected. The presence of fibers is most 
likely due to the combined contribution of both wastewater and runoff. The levels observed in CSOs are higher than the levels in strict separated runoff. For two of the three samples, concentrations were higher than the concentrations in wastewater. A settlement of particles during dry weather periods and their re-suspension when the flow increases was described on the literature [22]. A similar behavior could be expected for the fibers. The level of fibers also depends on the previous rain events: the first sampling was conducted after a long period of heavy rainfall, which might have induced a decrease in the amount of fibers in the sewer system.

Levels of fragments vary between 35 and 3,100 fragments $\mathrm{L}^{-1}$. The levels are especially high even if they vary by two orders of magnitude. Lower concentrations of fragments in comparison to fibers can be observed except for the event presenting the highest runoff contribution and volume.

\subsection{Fiber Fluxes in Different Compartments}

As fibers are of utmost importance concerning MP pollution in freshwater, it is especially interesting to assess the relative contribution of the various sources. In this section, the number of fiber fluxes is estimated at the scale of the Paris Megacity (surface area around 2,500 $\mathrm{km}^{2}-10$ million inhabitants). The number of MP fibers is used to estimate the mass fluxes. Because the fibers were measured, the cumulated total length of the fibers was calculated. The length was coupled with their approximated diameter to evaluate the volume. It was estimated that diameters ranged between 5 and $100 \mu \mathrm{m}$ with an average diameter of $25 \mu \mathrm{m}$. Therefore, with the total volume and specific densities of the plastic polymers $\left(1 \mathrm{~g} \mathrm{~cm}^{-3}\right.$ for the PA and $1.45 \mathrm{~g} \mathrm{~cm}^{-3}$ for the PET, corresponding to polymers widely used in the textile industry), total masses can be estimated. The results are summarized in Fig. 2.

- Atmospheric fallout. According to the average atmospheric flux of total fibers at the urban and suburban sites (110 and 53 fibers $\mathrm{m}^{-2}$ day $^{-1}$ ), we can estimate that at the scale of the Paris agglomeration between 1.2 and $2.5 \times 10^{11}$ fibers could originate annually from the atmosphere. According to our analyses, we calculate that $30 \%$ of these fibers are plastic polymers. Therefore, between 3.5 and $7.6 \times 10^{10}$ MPs would fall per year from the atmosphere on the Paris agglomeration. The masses of plastic fibers are likely between 6 and 17 metric tons.

- Gray water. Based on the water consumption for washing machine in France (14.4 $\mathrm{L} \mathrm{inhab}^{-1} \mathrm{day}^{-1}$ ) and 10 millions of inhabitants, we assess that between $4 \times 10^{14}$ and $2 \times 10^{15}$ fibers are discharged annually into the wastewater. However, the washing machine fibers were not chemically characterized in this work. Two hypotheses can be assumed. For the first one, we can consider that, at a global scale, $60 \%$ of these fibers are synthetic, according to the Europeans' uses and supposing also that both categories of fibers tear off similarly from clothes. The second hypothesis is based on a talk at the SETAC Europe 2016 conference indicating that MPs account for 5\% of the fibers at the 
disposal of washing machines [23]. Therefore, based on both scenarios (5 and $60 \%$ of synthetic fibers), between $2 \times 10^{13}$ and $1 \times 10^{15}$ annual MP fibers are discharged into the wastewater at the Paris agglomeration scale. This corresponds to a mass between 6 and 437 metric tons/year.

- Wastewater. As 2.3 million $\mathrm{m}^{3}$ wastewater are treated daily for the Paris agglomeration, between $6 \times 10^{13}$ and $4 \times 10^{14}$ fibers flow annually in the wastewater. Assuming that the proportion of synthetic fibers remains constant between the washing machine disposal and the entry of the wastewater treatment plant, it was considered that between 5 and $60 \%$ of the fibers in wastewater are synthetic. Therefore, between $3 \times 10^{12}$ and $3 \times 10^{14}$ synthetic fibers, i.e., between 2 and 225 metric tons of fibrous MPs, flow annually on wastewater. By applying the removal rates of WWTP Seine Centre (between 80 and 95\%) to the global estimation made above for the wastewater, we estimate that the Paris agglomeration releases annually between $2 \times 10^{11}$ and $5 \times 10^{13}$ plastic fibers into the surface waters, corresponding to a mass between 0.1 and 45 metric tons.

- Combined sewer overflows. CSO discharges in the Paris combined sewage system are approximated about 21 million $\mathrm{m}^{3}$ year ${ }^{-1}$, corresponding to a potential introduction into the freshwater of between 4 and $5 \times 10^{12}$ fibers annually. It is hard to provide an accurate estimation of the proportion of plastic polymers among those fibers.

\subsection{Comparison of Microplastic Sources in Freshwater}

Among the various sources investigated, fibers were always present, while fragments were mainly detected in the urban runoff and the CSOs. The atmospheric compartment was confirmed as a source of fibers including MPs. These fibers could have different sources including synthetic fibers from clothes and houses, degradation of macroplastics, landfills, or waste incineration. The characterization indicates that the hypothesis of the clothing being the main source of these fibers is the most plausible (proportion of polymers close to the uses on the textile industry). These fibers in the atmosphere, including MPs, could be transported by wind to the aquatic environment or deposited on surfaces of cities or agrosystems. After deposition, they could impact terrestrial organisms or be transported into the aquatic systems through runoff. Future work is needed in order to investigate these atmospheric fibers and understand where they come from, where they end up, and which mechanisms and factors lead to their transport and their fallout. The distance over which a fiber could be transported is also still unknown. In this study, it was not possible to assess whether the observed fibers come from very close sources in the proximity or from distant places. MPs found in isolated lakes suggest that the transport could occur over long distances [24]. It seems that atmospheric fallout is a significant source of MPs and should be considered when investigating MPs in freshwater. In addition to atmospheric fallout, other sources have to be considered like fibers that can deposit directly from the clothes of people walking on streets. In 
addition, fibers coming from dry weather atmospheric fallout could be re-suspended by wind or washed during the roads and streets cleaning, limiting the accumulation between two rain events.

While only fibers were detected in atmospheric fallout, runoff contained also fragments. It suggests that fibers can be transported by air, while the larger fragments seem to fall directly on surfaces and wait to be transported by the urban runoff, if not cleaned by road cleaning services. We can suppose that the fragments stem from the degradation of larger debris, but this hypothesis still needs verification.

WWTPs were also studied as potential sources of MPs. Fibers were found in washing machine effluents and consequently in wastewater. Mechanisms and dynamics that fibers undergo inside of a sewage system are up to now not reported in the literature. The roughly estimated flux of fibers entering WWTPs lies in the same range as the amounts supposedly discharged by washing machines. The estimation range is however large and fluxes could be potentially lower. Because the transport duration in the sewer systems is probably short (max. 48-72 h), it is considered that no fragmentation occurs. On the other hand, a sedimentation process during dry weather periods in the sewage network is possible. WWTP effluents are perhaps the most investigated sources to the receiving systems. For the different Parisian WWTPs, the estimated number of MP fibers even with a removal of fibers between 80 and $95 \%$ is higher than the fibers coming from atmospheric fallout. The WWTP effluents seem to be the major source of fibers in comparison to other MPs.

In contrast, CSOs contain both high fragments and fiber concentrations. It appears that CSOs are the main and major input of MP fragments into the freshwater. Moreover, the fact that it presents concentrations of fibers sometimes higher than wastewater tends to confirm a re-suspension of sewer deposits during wet weather periods.

The knowledge on the various dynamics and mechanisms of MPs in urban catchments is still very coarse. For instance, conditions driving the fibers suspension, the aerial transport, and the fallout processes are unknown. If some sources and fluxes have been identified, it is necessary to compare the results obtained on the Paris Megacity on other case studies all over the world.

\section{Monitoring Microplastics in the River Seine}

\subsection{Overview of the Approach}

In a preliminary study published at early stages of this work, we tested two different mesh size nets to sample the river Seine [18]. It highlighted the differences between a small $(80 \mu \mathrm{m})$ and a larger mesh size $(330 \mu \mathrm{m})$. Fibers are highly concentrated and 
the use of the $80 \mu \mathrm{m}$ mesh size is preferred. Fragments on the other hand are less abundant, and sampling higher volumes is mandatory, requiring large mesh size $(330 \mu \mathrm{m})$. In this chapter, both methods were employed. Moreover, because fibers seem to characterize the area of the case study and are less investigated by the previously published investigations on freshwater, a long-term monitoring from April 2014 to December 2015 was carried out on four stations (P2-P5) on the Seine River from upstream to downstream Paris plus one station on the Marne River (P1). For the fragments, only five different campaigns were carried out on the various sites (Fig. 1).

\subsection{Fibers in the Seine and Marne Rivers}

Concentrations through the year in the Marne River (P1) range between 5.7 and 398.0 fibers $\mathrm{m}^{-3}$ with a mean concentration of $100.6 \pm 99.9$ fibers $\mathrm{m}^{-3}$ (mean $\pm \mathrm{SD}$ ). From the upstream to the downstream points, the concentrations are $48.5 \pm 98.5$ fibers $\mathrm{m}^{-3}(\mathrm{P} 2), 27.9 \pm 26.3$ fibers $\mathrm{m}^{-3}(\mathrm{P} 3), 27.9 \pm 40.3$ fibers $\mathrm{m}$ $-3(\mathrm{P} 4)$, and $22.1 \pm 25.3$ fibers $\mathrm{m}^{-3}$. Variations occurred in a parallel way on the different sites. This could indicate that global factors that vary equally for all sites are more likely to affect the concentrations than local factors. The variations in diffusive inputs or seasonal changes could be at cause. We could also suspect a relation with the river flow variations, but no clear correlation was found. Nonetheless, a tendency to always have low fiber levels during high-water-flow periods was observed. During low-water-flow periods, levels are much more variable and could be influenced by different parameters such as the input of fibers, either from punctual sources (WWTP, CSO), diffusive sources (atmospheric fallout), or a possible re-suspension of fibers from the sediments.

It is possible to assess the annual fluxes of fibers in the Seine River using the 19 punctual fluxes calculated at each site. The increase between the most upstream and most downstream point (P2 and P5) is only 6\%, which is much smaller than the uncertainty induced by the short-term variability (unpublished data). As a consequence, it seems regarding the fibers that the impact of the Paris agglomeration is rather small. Current state of knowledge does not allow to understand and explain this non-increasing pattern. In fact, between P2 and P5, two tributaries (Marne and Oise Rivers), three WWTP disposals (Seine Amont, Seine Centre, and Seine Aval), and numerous CSOs join the Seine River. Sinks that counterbalance these inputs could explain the fact that similar fluxes are found from upstream and downstream Paris. We suspect an important role related to the sedimentation and deposition on the banks of the fibers. Further research on the fate of the fibers is still required.

The minimum and maximum estimated fluxes for the site P5 are $2.8 \times 10^{10}$ and $6.1 \times 10^{11}$ fiber/year with a mean of $1.8 \times 10^{11}$. With the hypothesis that $65 \%$ of the fibers are synthetic, we approximate that between $1.8 \times 10^{10}$ and $4.0 \times 10^{11} \mathrm{MP}$ 
fibers flow in 1 year in this site corresponding to an estimated mass of synthetic fibers between 0.01 and 0.34 metric tons flow per year. The evolution of this flux toward the estuary has to be determined in order to be able to determine the input from freshwater to the marine environment in terms of MPs.

\subsection{Comparison with the Fragments}

There is a large difference in the concentration levels between fibers and fragments. The mean fiber concentration is around 45 fibers $\mathrm{m}^{-3}$ ( $n=95$ samples), while the mean fragment concentrations considering both methods is around 0.54 fragments $\mathrm{m}^{-3}$ ( $n=17$ samples). As a consequence, using two different sampling methods for fibers and fragments seems really pertinent. While analyzing fibers needs the use of a small mesh size, sampling higher volumes is mandatory to collect other shapes of MPs.

By assuming a mean fiber length $(973 \mu \mathrm{m})$ and diameter $(25 \mu \mathrm{m})$ and for the fragments the mean area $(168,000 \mu \mathrm{m}$ [2]) and roughly estimated thickness $(35 \mu \mathrm{m})$, the volumes of a typical particle for each shape can be approximated. Combining MP proportions and polymer densities hypotheses $\left(1\right.$ and $\left.1.45 \mathrm{~g} \mathrm{~cm}^{-3}\right)$, the mass concentrations were approached.

It was estimated that the mean concentration for synthetic fibers is of $2 \times 10^{-5}$ $\mathrm{g} \mathrm{m}^{-3}$, while it is of $3 \times 10^{-6} \mathrm{~g} \mathrm{~m}^{-3}$ for fragments. Because of the small amount of data, the fragment mass flux was not estimated. However, it seems with this result that even if a fragment is bigger than a fiber on average, the fragment mass fluxes would be one order of magnitude smaller than fiber mass fluxes.

\section{Conclusions and Perspectives}

Although information on MPs in freshwater increased dramatically over the very recent years, there is, until now, neither a systematic overview of the sources, fate, and sinks on a catchment scale nor a link between the catchment characteristics and the concentration of MPs in receiving systems.

A first attempt was made on the urban catchment of Paris Megacity and its main drainage system: the Seine and the Marne Rivers. During almost 3 years, samples have been collected from atmospheric fallouts and urban runoff, from upstream to downstream of the sewage system, and in the rivers. The key results are:

- The importance of the fiber category (near urban areas at least), which includes not only plastic fibers but also other synthetic fibers like rayon, which might also have an environmental impact. 
- The necessity to split MPs in two categories: fibers and fragments. Sampling protocols often do not allow the sampling of fibers. This study shows that microplastic fibers are numerous in the catchment and in the receiving system and cannot be neglected. Of course the impact of such fibers is still unknown and must be investigated. Fragments are two orders of magnitude less abundant than fibers, but with different environmental impacts and must also be analyzed.

- Atmospheric fallouts constitute a significant flux of fibers at the scale of an urban catchment. Up to now, the dynamics of these fibers in the atmosphere, its interaction with the catchment surface, the alternating mechanisms of fallout/ re-suspension, and the length scale of the movement of a fiber in the atmosphere remain unknown. Moreover, while studies point out at the rivers as a major introduction way of the fibers from the continental into the marine environment, this study suggests that the atmospheric compartment and the wind should be further investigated as a potentially major contributor.

- Washing machines seem to be a major source of fibers, including MP fibers. However, wastewater treatment plants play a major role in the reduction of fibers and microplastic fragments, which are probably transferred to sludge. Investigation of sludges and, when sludge spreading takes place, of agricultural land has still to be reinforced.

- Sampling protocol of fragments in rivers has to be improved, in order to decrease the uncertainty in downstream flow estimates.

- Concerning fibers, surprisingly no obvious increase of the upstream-downstream flow has been observed. Various in-river phenomena may explain this, but both in situ measurements and lab experiments have to be conducted to clarify this point.

Actually, this study is only the starting point of a more comprehensive work related to the dynamics of (micro)plastics at a catchment and especially urban catchment scale. Other places have to be investigated and consistent interdisciplinary research programs conducted. This study showed mainly that the identification of the sources and the fate of microplastic in freshwater is complicated and that a systematic and holistic approach is required.

Acknowledgments The authors acknowledge all the partners who collaborated during this work. We thank the technical teams of SIAAP; those of the Val-de-Marne General Council; Anne Chabas of the Interuniversity Laboratory of Atmospheric Systems (LISA); Emmanuel Rinner and Kada Boukerma of the IFREMER Detection, Sensors, and Measurements Laboratory; and the Bruker demonstrating laboratory in Champs-sur-Marne and its staff. This work is part of a thesis funded by the Paris research network on sustainable development (R2DS Ile-de-France). 


\section{References}

1. Carpenter EJ, Anderson SJ, Harvey GR, Miklas HP, Peck BB (1972) Polystyrene spherules in coastal waters. Science 178:749-750

2. Carpenter EJ, Smith KL (1972) Plastics on the Sargasso Sea surface. Science 175:1240-1241

3. Thompson RC, Olsen Y, Mitchell RP, Davis A, Rowland SJ, John AWG et al (2004) Lost at sea: where is all the plastic? Science 304:838-838

4. Dris R, Imhof H, Sanchez W, Gasperi J, Galgani F, Tassin B et al (2015) Beyond the ocean: contamination of freshwater ecosystems with (micro-)plastic particles. Environ Chem 12:539

5. Arthur C, Baker J, Bamford H (2008) In: Arthur C, Baker J, Bamford H (eds) Proceedings of the international research workshop on the occurrence, effects, and fate of microplastic marine Debris. National Oceanic and Atmospheric Administration: University of Washington Tacoma campus in Tacoma, Washington. http://marinedebris.noaa.gov/sites/default/files/ Microplastics.pdf

6. Zbyszewski M, Corcoran PL (2011) Distribution and degradation of fresh water plastic particles along the beaches of Lake Huron. Can Water Air Soil Pollut 220:365-372

7. Moore CJ, Lattin GL, Zellers AF (2011) Quantity and type of plastic debris flowing from two urban rivers to coastal waters and beaches of southern. J Integr Coastal Zone Manage 11:65-73

8. Wilber RJ (1987) Plastic in the North Atlantic. Oceanus 30:61-68

9. Gregory MR (1996) Plastic 'scrubbers' in hand cleansers: a further (and minor) source for marine pollution identified. Mar Pollut Bull 32:867-871

10. Fendall LS, Sewell MA (2009) Contributing to marine pollution by washing your face: microplastics in facial cleansers. Mar Pollut Bull 58:1225-1228

11. Cooper DA, Corcoran PL (2010) Effects of mechanical and chemical processes on the degradation of plastic beach debris on the island of Kauai. Hawaii Mar Pollut Bull 60:650-654

12. Gregory MR, Andrady AL (2003) Plastics in the marine environment. In: Andrady AL (ed) Plastics and the environment. Wiley, Hoboken, pp 379-401. doi:10.1002/0471721557. ch10/summary

13. Browne MA, Crump P, Niven SJ, Teuten E, Tonkin A, Galloway T et al (2011) Accumulation of microplastic on shorelines worldwide: sources and sinks. Environ Sci Technol 45: 9175-9179

14. Plastics Europe (2015) Plastics - the Facts 2015, an analysis of European latest plastics production, demand and waste data. Plastics Europe, Association of Plastic Manufacturers, Brussels, $\mathrm{p} 40$

15. Industrievereinigung Chemiefaser, Chemical fiber global production by type 2000-2014 statistic [internet]. Statista 2015 [cited 20 Jun 2016]. http://www.statista.com/statistics/ 271651/global-production-of-the-chemical-fiber-industry/

16. Dris R (2016) First assessment of sources and fate of macro- and micro-plastics in urban hydrosystems: case of Paris megacity. PhD thesis, LEESU, Paris

17. Dris R, Gasperi J, Saad M, Mirande C, Tassin B (2016) Synthetic fibers in atmospheric fallout: a source of microplastics in the environment? Mar Pollut Bull 104:290-293

18. Dris R, Gasperi J, Rocher V, Saad M, Renault N, Tassin B (2015) Microplastic contamination in an urban area: a case study in Greater Paris. Environ Chem 12:592-599

19. Talvitie J, Heinonen M, Paakkonen J-P, Vahtera E, Mikola A, Setala O et al (2015) Do wastewater treatment plants act as a potential point source of microplastics? Preliminary study in the coastal Gulf of Finland, Baltic Sea. Water Sci Technol 72:1495-1504

20. Murphy F, Ewins C, Carbonnier F, Quinn B (2016) Wastewater treatment works (WwTW) as a source of microplastics in the aquatic environment. Environ Sci Technol 50:5800-5808

21. Carr SA, Liu J, Tesoro AG (2016) Transport and fate of microplastic particles in wastewater treatment plants. Water Res 91:174-182 
22. Gasperi J, Garnaud S, Rocher V, Moilleron R (2009) Priority pollutants in surface waters and settleable particles within a densely urbanised area: case study of Paris (France). Sci Total Environ 407:2900-2908

23. Llorca M, Schirinzi G, Sanchis J, Barcelo D, Marinella F (2016) Evaluation of the analysis of microplastics by mass spectrometry and assessment of their adsorption capacity for organic contaminants. SETAC Europe Nantes

24. Free CM, Jensen OP, Mason SA, Eriksen M, Williamson NJ, Boldgiv B (2014) High-levels of microplastic pollution in a large, remote, mountain lake. Mar Pollut Bull 85:156-163

Open Access This chapter is licensed under the terms of the Creative Commons Attribution 4.0 International License (http://creativecommons.org/licenses/by/4.0/), which permits use, sharing, adaptation, distribution and reproduction in any medium or format, as long as you give appropriate credit to the original author(s) and the source, provide a link to the Creative Commons license and indicate if changes were made.

The images or other third party material in this chapter are included in the chapter's Creative Commons license, unless indicated otherwise in a credit line to the material. If material is not included in the chapter's Creative Commons license and your intended use is not permitted by statutory regulation or exceeds the permitted use, you will need to obtain permission directly from the copyright holder. 